\title{
Metabolic Tumor Volume Predicts Disease Progression and Survival in Patients with Squamous Cell Carcinoma of the Anal Canal
}

\author{
Jose G. Bazan ${ }^{1}$, Albert C. Koong ${ }^{1}$, Daniel S. Kapp ${ }^{1}$, Andrew Quon ${ }^{2}$, Edward E. Graves ${ }^{1}$, Billy W. Loo, Jr. ${ }^{1}$, \\ and Daniel T. Chang ${ }^{1}$ \\ ${ }^{1}$ Department of Radiation Oncology, Stanford University, Stanford, California; and ${ }^{2}$ Department of Radiology, \\ Division of Nuclear Medicine, Stanford University, Stanford, California
}

PET imaging has become a useful diagnostic tool in patients with anal cancer. We evaluated the prognostic value of metabolic tumor volume (MTV) in patients with anal cancer treated with definitive chemoradiotherapy. Methods: Patients with anal cancer who underwent PET imaging for pretreatment staging or radiation therapy planning from 2003 to 2011 were included. PET parameters included MTV and maximum standardized uptake value (SUVmax). Total MTV (MTV-T) was defined as the sum of the volumes above a standardized uptake value $50 \%$ of the SUVmax within the primary tumor and involved nodes. KaplanMeier and Cox regression models were used to test for associations between metabolic or clinical endpoints and overall survival (OS), progression-free survival (PFS), and event-free survival (EFS). Results: Thirty-nine patients were included. Median follow-up for the cohort was 22 mo. Overall, 6 patients died and 9 patients had disease progression. The 2-y OS, PFS, and EFS for the entire cohort were $88 \%, 74 \%$, and $69 \%$, respectively. Higher MTV-T was associated with worse OS $(P=0.04)$, PFS $(P=0.004)$, and EFS $(P=0.002)$ on univariate analysis. Patients with an MTV greater than $26 \mathrm{~cm}^{3}$ had worse PFS than did those with an MTV of $26 \mathrm{~cm}^{3}$ or less (33\% vs. $82 \%, P=0.003$ ). SUVmax was not prognostic for any outcome. Higher T classification (T3/T4 vs. T1/T2) was associated with worse PFS and EFS. When adjusting for T classification, MTV-T remained a significant predictor for PFS $(P=0.01)$ and EFS $(P=0.02)$. Conclusion: MTV-T yields prognostic information on PFS and EFS beyond that of established prognostic factors in patients with anal cancer.

Key Words: PET; metabolic tumor volume; anal cancer; IMRT; SUV

J Nucl Med 2013; 54:27-32

DOI: 10.2967/jnumed.112.109470

C treatment paradigm for squamous cell carcinoma of the

\footnotetext{
Received May 30, 2012; revision accepted Aug. 9, 2012.

For correspondence or reprints contact: Daniel T. Chang, Department of Radiation Oncology, Stanford University Medical Center, 875 Blake Wilbur Dr., Stanford, CA 94305-5847.

E-mail: dtchang@stanford.edu

Published online Dec. 12, 2012.

COPYRIGHT (C 2013 by the Society of Nuclear Medicine and Molecular Imaging, Inc.
}

anal canal (SCC-AC) on the basis of several randomized trials (1-4). Disease outcomes for SCC-AC are generally more favorable than for other gastrointestinal sites, but the 5 -y disease-free survival (DFS) rates in randomized trials have been $60 \%-65 \%(1,2)$ indicating a need to identify pretreatment prognostic factors for failure to help to individualize treatment in an effort to improve the outcomes in high-risk patients. A recently updated analysis of Radiation Therapy Oncology Group (RTOG) 98-11 firmly established tumor diameter as an important prognostic marker for DFS and overall survival (OS) (5).

Recently, PET has become a valuable tool in the staging, treatment, and surveillance of patients with various malignancies. The use of PET to stage anal cancer is increasing as a result of several studies (6-9). There are few reports, however, regarding the utility of PET as a prognostic marker in patients with anal cancer. Kidd et al. demonstrated that higher baseline maximum standardized uptake value (SUVmax) in the primary anal tumor predicted worse DFS (10). Two other groups found that posttreatment metabolic response (determined by change in SUVmax) predicted PFS $(11,12)$.

Although these studies have focused on SUVmax, a growing body of literature supports that the metabolic tumor volume (MTV) is a valuable biomarker for patients with malignancies from multiple sites, including lung $(13,14)$, head and neck (15-17), esophagus (18), and pancreas (19). MTV reflects the volume of tumor tissue that demonstrates increased hypermetabolic activity on PET and may thereby more accurately represent a patient's tumor burden. MTV has been shown to be a more important prognosticator than SUVmax and stage $(13,17)$. The purpose of the current study was to consider the role of MTV and SUV at diagnosis in patients with SCC-AC treated with chemoradiotherapy.

\section{MATERIALS AND METHODS}

\section{Patients}

In this study approved by the institutional review board, we identified 47 patients in our departmental database who had been treated with pelvic intensity-modulated radiation therapy (IMRT) for histologically proven SCC-AC between 2003 (the year our institution began treating these patients with IMRT) and 2011. 
Patients were included in this retrospective study if they underwent PET either for staging or for radiation therapy planning and if they received definitive chemoradiotherapy (curative treatment for nonmetastatic disease). Those patients who were excluded included 6 who did not undergo pretherapy PET/CT and 2 who had metastatic disease at the time of disease presentation. The remaining 39 patients formed the cohort for this study.

\section{Treatment}

Treatment consisted of chemoradiotherapy with IMRT. Our methods for patient simulation and target volume delineation have been described previously (20). Briefly, we treated the low-risk (common iliac, proximal internal iliac superior to the inferior aspect of the sacroiliac joint, presacral, external iliac, and uninvolved inguinal nodes) planning target volume to $40 \mathrm{~Gy}$ at $1.6 \mathrm{~Gy}$ per fraction, the intermediate-risk planning target volume (internal iliac inferior to the inferior aspect of the sacroiliac joint, involved inguinal and perirectal nodal regions) to 45 Gy at 1.8 Gy per fraction, and the high-risk planning target volume (primary tumor and gross nodal disease) to $45 \mathrm{~Gy}$ with a sequential boost of $5.4 \mathrm{~Gy}$ for T1-T2 tumors and 9-14.4 Gy for T3-T4 tumors and grossly positive nodes.

All patients received 5-flourouracil (5FU)-based chemotherapy (20 received capecitabine and 19 infusional 5FU). Thirty-four patients (87\%) received mitomycin-C (MMC) plus 5FU, 4 patients (10\%) received concurrent platinum plus $5 \mathrm{FU}$ (3 received cisplatin and 1 carboplatin), and 1 patient (3\%) received 5FU alone.

\section{PET Protocol}

All scans were acquired on a Discovery LS PET/CT scanner (GE Healthcare). PET images were analyzed as previously described according to an institutional protocol (13). Briefly, each patient fasted for at least $8 \mathrm{~h}$ before imaging, and the blood glucose levels of all patients were measured before the injection of ${ }^{18}$ F-FDG. The average blood glucose level was $100 \pm 19 \mathrm{mg} / \mathrm{dL}$. Patients were injected with $259-629 \mathrm{MBq}$ of ${ }^{18}$ F-FDG at $71 \pm$ $18 \mathrm{~min}$ before image acquisition.

The field of view covered the scalp to mid femur in 25 patients who underwent diagnostic scans and from the upper abdomen to mid femur in 14 patients who underwent radiation therapy planning scans. PET data were acquired in 2-dimensional mode for 3-5 min per bed position. The PET slice thickness was $3.27 \mathrm{~mm}$. Helical CT images were obtained with the following acquisition parameters: $120 \mathrm{kVp}, 220 \mathrm{mAs}$, 1-s rotation, 20-mm beam collimation, 1.675 pitch, and $10 \mathrm{~mm} / \mathrm{s}$ bed speed. The PET data were then reconstructed with an ordered-set expectation maximization algorithm, using the CT images for attenuation correction. CT images were reconstructed using a slice thickness of $2.5 \mathrm{~mm}$ every $3.27 \mathrm{~mm}$. Reconstructed data images were then transferred to a radiation treatment planning workstation and to a research workstation for further analysis.

\section{Measurement of Hypermetabolic Tumor Volume: Fixed Percentage Threshold Segmentation}

Computer-aided MTV and standardized uptake value (SUV) measurements were performed using RT_Image, an open-source software package developed at our institution, as described previously $(13,14,16,19,21)$. A maximum-intensity-projection view of the images permitted rapid visual identification of the hypermetabolic lesions. The MTV was defined as the volume of hypermetabolic tissue with a threshold of greater than $50 \%$ of the SUVmax in the primary site (MTV-P) and involved lymph nodes (MTV-N). Although there is currently no uniformly accepted definition for
MTV, the threshold of $50 \%$ was chosen on the basis of an early phantom study that identified the $50 \%$ intensity line as a reasonable choice (22). In addition, numerous studies in a variety of disease sites have demonstrated that MTV as defined with the 50\% threshold definition is significantly correlated with patient outcome (13-17). Figure 1 demonstrates an example of the MTV-P for a patient in our study. Once all hypermetabolic foci for each patient were identified, the total MTV (MTV-T) was calculated by the software as the sum of MTV-P and MTV-N. The MTV-T, MTV-P, and MTV-N were viewed in the axial, coronal, and sagittal planes to ensure that normal tissues were not incorporated. The software also calculated the SUVmax and mean SUV within the MTVs. The total glycolytic activity (TGA) was defined as the (mean SUV) $\times($ MTV-T). Peak SUV (SUVpeak) was calculated by the software according to the RTOG 0235 protocol as the mean SUV within a 1-cm-diameter circle centered on the SUVmax (23). These parameters were retrospectively generated for this analysis and did not influence the radiation prescription dose at the time of treatment.

\section{Follow-up}

The patients were seen by their radiation oncologist at least once weekly during the course of treatment. After completing therapy, they were followed clinically every 2-3 mo for year 1 , generally alternating visits between radiation oncology, medical oncology, and general surgery. One patient was lost to follow-up after the first clinical examination. Imaging studies were performed for 34 of the 39 patients at 3-4 mo after chemoradiotherapy including 23 posttherapy PET scans and 11 posttherapy diagnostic CT scans of the chest, abdomen, and pelvis. The remaining patients underwent their first surveillance scan more than 6 mo after completion of therapy. They were then examined every 3-4 mo during years 2-3 and every 6 mo after year 3 .

\section{Statistical Analysis}

Data were analyzed using SPSS Statistics (version 19; IBM Corp.) and SAS (version 9.3; SAS Institute). Actuarial rates were estimated using the Kaplan-Meier method. OS was calculated from the date of treatment initiation to the date of death from any cause. Progressionfree survival (PFS) was calculated as the interval from the date of treatment initiation to the date of the first finding on imaging or examination indicating local or distant disease progression that led

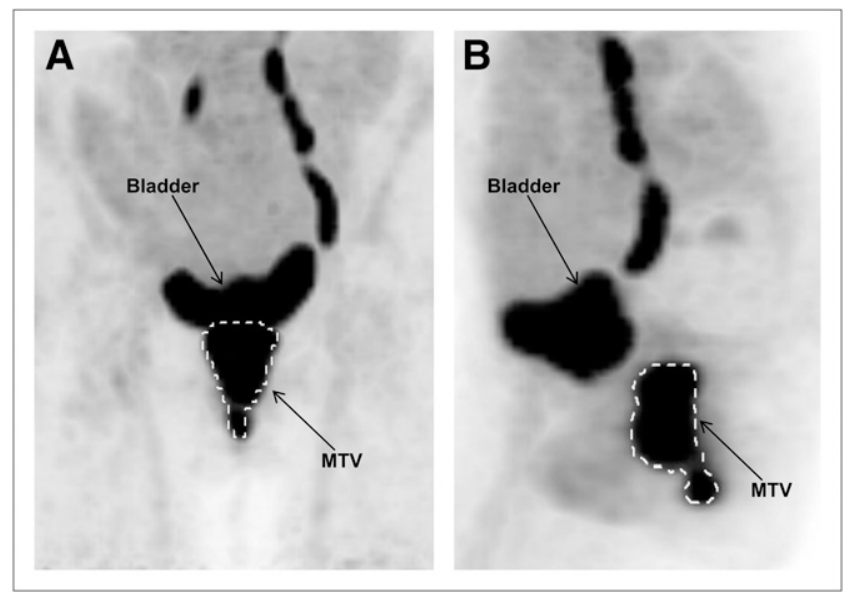

FIGURE 1. Representative PET maximum-intensity-projection images with MTV represented by dashed line on coronal $(A)$ and sagittal (B) images. Sagittal image demonstrates clearer separation between tumor and bladder. 
to additional confirmatory testing or death due to disease (including treatment-related deaths). Event-free survival (EFS) included patients who met either the OS or the PFS endpoints. The level of significance was estimated using the log-rank test. Time-dependent receiver-operating-characteristic analysis was used to determine area under the curve to estimate the predictive ability of MTV-T and SUVmax (24). Cox regression analysis was used to evaluate prognostic variables for univariate and multivariate predictions of OS, PFS, and EFS. The prognostic factors that were analyzed included the continuous variables MTV, SUVmax, SUVpeak, and age and the categoric variables sex (female vs. male), HIV status (HIV-positive vs. HIV-negative), MMCbased chemotherapy (yes vs. no), and T classification (T1-T2 vs. T3-T4). Tests were based on the likelihood-ratio statistic. The Pearson correlation coefficient was used to test associations between continuous variables as appropriate.

\section{RESULTS}

\section{Patient and Tumor Characteristics}

The characteristics of the 39 patients in this study are summarized in Table 1. Most of the patients were female, and $10 \%$ were HIV-positive. The median time between the PET scan and the initiation of treatment was $14 \mathrm{~d}$ (mean, $15 \mathrm{~d}$; range, 5-41 d). Median radiation dose to the primary tumor was 54 Gy (range, 45-59.4 Gy).

The median MTV-T was $9 \mathrm{~cm}^{3}$ (range, $0.1-120 \mathrm{~cm}^{3}$ ), and the median TGA was $55 \mathrm{~cm}^{3}$ (range, $0.3-1038 \mathrm{~cm}^{3}$ ). Patients with T3/T4 tumors tended to have larger MTV-T than those with T1/T2 tumors (median, 15 vs. $6 \mathrm{~cm}^{3}$ ), but there was a significant overlap in MTV-T between the 2 groups (Fig. 2). Although MTV-T did not influence radiation dose prescription, patients with an MTV-T of $9 \mathrm{~cm}^{3}$ or less on average received a lower radiation dose than did patients with an MTV-T greater than $9 \mathrm{~cm}^{3}$ (median, 50.4 vs. 54

TABLE 1

Patient Characteristics

\begin{tabular}{|cc}
\hline Characteristic & $n$ \\
\hline Sex & \\
\hline Female & 24 \\
Male & 15 \\
HIV-positive & $4(10)$ \\
T class & \\
\hline T1 & $6(15)$ \\
T2 & $20(51)$ \\
T3 & $10(26)$ \\
T4 & $3(8)$ \\
N class & $27(70)$ \\
N0 & $6(15)$ \\
N1 & $4(10)$ \\
N2 & $2(5)$ \\
N3 & \\
Chemotherapy & 34 \\
MMC/5FU & 4 \\
Cisplatin-based & 1 \\
\hline 5FU alone & \\
&
\end{tabular}

Median patient age was $58 \mathrm{y}$, and age range was $40-87 \mathrm{y}$. Data in parentheses are percentages.

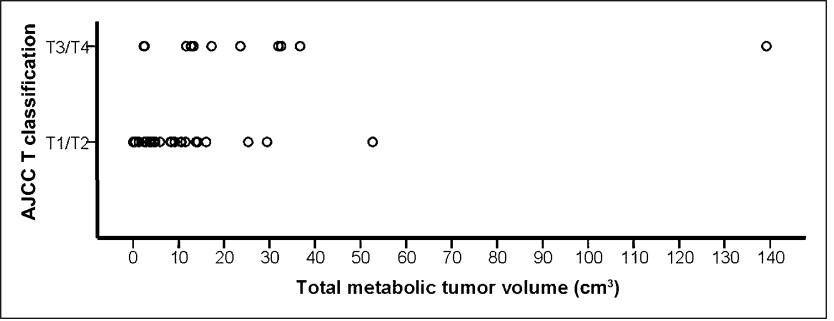

FIGURE 2. MTV-T by T classification of American Joint Committee on Cancer (AJCC).

Gy). Still, 47\% (9/19) of patients with an MTV-T of $9 \mathrm{~cm}^{3}$ or less received 54-59.4 Gy.

Median values for SUVmax and SUVpeak were 11.7 (range, 3.8-32.8) and 10.1 (range, 2.9-32.8), respectively. There was significant overlap in SUVmax and SUVpeak among patients with T1/T2 tumors and those with T3/T4 tumors.

\section{Prognostic Value of PET Parameters}

At a median follow-up of 22 mo (range, 2-82 mo), 6 patients had died: 2 deaths from disease, 1 treatment-related death, and 3 deaths without documented recurrence at last follow-up ( 1 stroke, 1 alcoholic liver failure, and 1 neurodegenerative disease). Nine patients experienced disease progression, including 4 local failures and 6 distant failures (1 patient with both). The numbers of analyzable events for OS, PFS, and EFS were 6, 10, and 13, respectively. The 2-y OS, PFS, and EFS rates were $88 \%, 74 \%$, and $69 \%$, respectively. Progression occurred in all T classifications (T1, $n=$ 2 ; T2, $n=3$; T3, $n=4$; and T4, $n=1$ ) and in 3 patients with nodal disease. Crude local and distant failure rates were $5 \%$ and $0 \%$, respectively, in patients with an MTV-T of $9 \mathrm{~cm}^{3}$ or less, compared with $15 \%$ and $30 \%$ in patients with an MTV-T greater than $9 \mathrm{~cm}^{3}$.

On univariate analysis, MTV-T and TGA were strong predictors of outcome. An increase in MTV-T of $12.5 \mathrm{~cm}^{3}$ (the interquartile range) was significantly associated with increased hazard ratios (HR) for OS (HR, 1.28; $P=0.04)$, PFS (HR, $1.5 ; P=0.002)$, and EFS (HR, $1.41 ; P=0.004)$ (Table 2). Similarly, an increase in the interquartile range for TGA was associated with a trend toward worse OS (1.53, $P=0.053)$ and significantly worse PFS $(1.76, P=0.006)$ and EFS $(1.61, P=0.016)$. Given the expected high degree of correlation between MTV-T and TGA $(r=0.95, P<2.5 \times$ $10^{-20}$ ) and the strong association between MTV-T and all 3 outcomes, subsequent analysis is based on MTV-T only.

The area under the curve for predicting PFS at $2 \mathrm{y}$ from MTV-T and SUVmax were 0.804 and 0.545 , respectively, indicating that MTV-T had a better predictive value than SUVmax. Using a cutoff point of $26 \mathrm{~cm}^{3}$ obtained from the receiver-operating-characteristic analysis, Figure 3 demonstrates that, compared with patients with an MTV-T of 26 $\mathrm{cm}^{3}$ or less, patients with an MTV-T greater than $26 \mathrm{~cm}^{3}$ had significantly worse PFS (33\% vs. $82 \%, P=0.003)$ and EFS (33\% vs. $76 \%, P=0.028)$, with a nonsignificant trend toward worse OS $(66.7 \%$ vs. $92.7 \%, P=0.367)$. 
TABLE 2

Cox Univariate Analysis

\begin{tabular}{|c|c|c|c|c|c|c|}
\hline \multirow[b]{2}{*}{ Parameter } & \multicolumn{2}{|c|}{ OS } & \multicolumn{2}{|c|}{ PFS } & \multicolumn{2}{|c|}{ EFS } \\
\hline & HR & $P$ & HR & $P$ & $\mathrm{HR}$ & $P$ \\
\hline Age (per y) & 1.06 & 0.17 & 1.02 & 0.48 & 1.03 & 0.28 \\
\hline Sex (female vs. male) & 0.467 & 0.41 & 2.49 & 0.26 & 1.45 & 0.55 \\
\hline T stage (T3/T4 vs. T1/T2) & 5.48 & 0.07 & 5.01 & 0.02 & 4.11 & 0.02 \\
\hline Nodal status (N-positive vs. N-negative) & 1.74 & 0.55 & 1.51 & 0.56 & 1.48 & 0.52 \\
\hline HIV status (HIV-positive vs. HIV-negative) & 1.92 & 0.56 & 0.881 & 0.91 & 0.662 & 0.69 \\
\hline MMC-based chemotherapy (yes vs. no) & 0.092 & 0.02 & 0.67 & 0.71 & 0.38 & 0.22 \\
\hline Time between PET and treatment $(\mathrm{d})$ & 0.95 & 0.49 & 1.048 & 0.23 & 1.022 & 0.58 \\
\hline MTV-T (per cm³) & 1.02 & 0.04 & 1.03 & 0.004 & 1.03 & 0.002 \\
\hline MTV-P & 1.02 & 0.048 & 1.04 & 0.001 & 1.03 & 0.004 \\
\hline MTV-N & 1.16 & 0.029 & 1.20 & 0.015 & 1.19 & 0.019 \\
\hline TGA (per cm³) & 1.003 & 0.053 & 1.004 & 0.006 & 1.003 & 0.02 \\
\hline SUVmax (per unit) & 1.00 & 0.99 & 0.990 & 0.85 & 1.003 & 0.95 \\
\hline SUVmax-P & 1.00 & 0.98 & 0.99 & 0.84 & 1.00 & 0.96 \\
\hline SUVmax-N & 1.08 & 0.33 & 1.08 & 0.23 & 1.06 & 0.23 \\
\hline SUVpeak (per unit) & 0.954 & 0.60 & 1.04 & 0.35 & 1.03 & 0.47 \\
\hline SUVpeak-P & 0.96 & 0.68 & 1.01 & 0.94 & 1.01 & 0.93 \\
\hline SUVpeak-N & 1.08 & 0.40 & 1.10 & 0.18 & 1.07 & 0.27 \\
\hline SUVmean (per unit) & 0.960 & 0.73 & 0.900 & 0.28 & 0.933 & 0.39 \\
\hline
\end{tabular}

SUVmax (HR, 0.99-1.003; $P>0.85$ ), SUVpeak (HR, 0.95-1.04; $P>0.35$ ), and SUVmean (HR, 0.90-0.96; $P>$ 0.28 ) were not associated with OS, PFS, or EFS (Table 2). T classification was significantly associated with PFS (HR, 5.01; $P=0.018$ ) and EFS (HR, 4.11; $P=0.016$ ), and MMC-based chemotherapy was associated with OS (HR, $0.09 ; P=0.02$ ). The time between PET scanning and treatment initiation was not correlated with any outcome (Table 2).

We further explored the subcomponents of MTV-T to determine whether either MTV-P or MTV-N was the main component of the association of MTV-T and outcome. Both MTV-P and MTV-N were significant predictors of all 3 outcomes (Table 2). Although the $P$ values for MTV-P were more significant for PFS and EFS than those of MTV-N, MTV-N appeared to be slightly more prognostic for OS.

We performed a similar analysis with regard to SUVmax and SUVpeak within the primary and nodal MTVs (Table 2).
There were weak trends toward associations between SUVmax-N/PFS $(P=0.23)$; SUVmax-N/EFS $(P=0.29)$; SUVpeak-N/PFS $(P=0.18)$; and SUVpeak-N/EFS $(P=0.27)$.

\section{MTV-T as Independent Prognostic Factor}

A multivariate analysis was undertaken as a purely exploratory maneuver to determine whether the univariate results for MTV-T were an artifact of confounders. We tested the association between MTV-T and outcome while controlling for each covariate individually (Table 3). When adjusting for T classification, MTV-T remained prognostic for PFS and EFS but not for OS. MTV-T remained an independent prognostic marker for PFS and EFS when adjusted for every other variable in the model, and it was prognostic for OS when adjusting for age, chemotherapy, and the various SUV metrics. T classification did not reach statistical significance for OS, PFS, or EFS when adjusting for MTV.
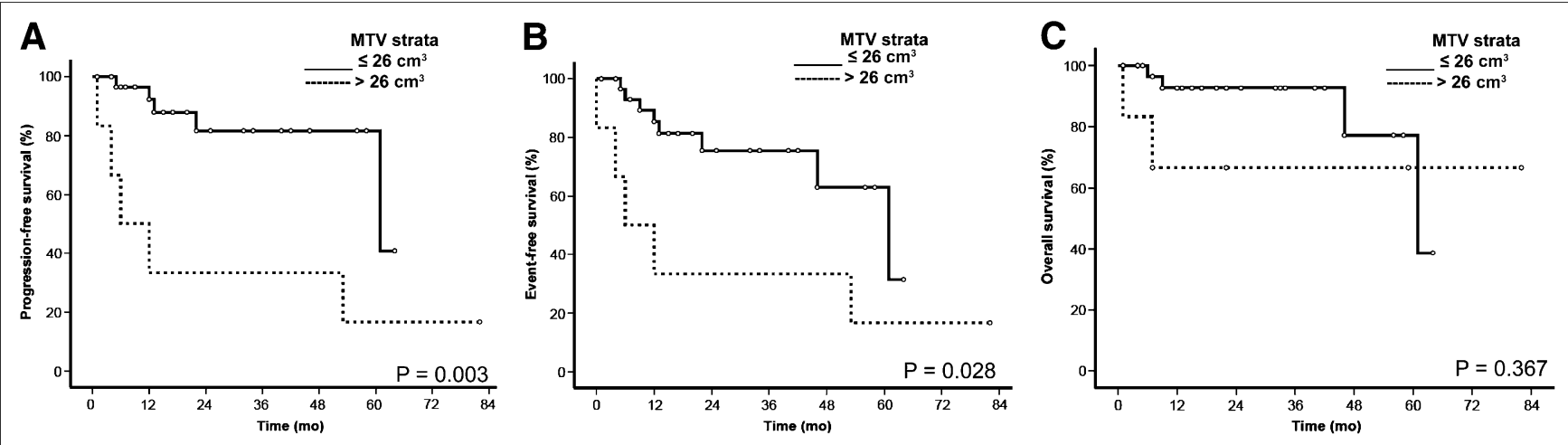

FIGURE 3. Kaplan-Meier analysis of PFS (A), EFS (B), and OS (C) by MTV-T. Patients were separated into 2 groups based on receiveroperating-characteristic analysis. Solid line = patients with MTV $\leq 26 \mathrm{~mL}$; dotted line = patients with MTV $>26 \mathrm{~mL}$; $\bigcirc=$ censored individuals. 
TABLE 3

Cox Multivariate Analysis

\begin{tabular}{|c|c|c|c|c|c|c|}
\hline \multirow[b]{2}{*}{ Parameter } & \multicolumn{2}{|c|}{ OS } & \multicolumn{2}{|c|}{ PFS } & \multicolumn{2}{|c|}{ EFS } \\
\hline & HR & $P$ & $\mathrm{HR}$ & $P$ & $\mathrm{HR}$ & $P$ \\
\hline MTV-T (per $\mathrm{mL}$ ) & 1.014 & 0.19 & 1.028 & 0.01 & 1.024 & 0.022 \\
\hline $\mathrm{T}$ class $(\mathrm{T} 3 / \mathrm{T} 4$ vs. $\mathrm{T} 1 / \mathrm{T} 2)$ & 4.13 & 0.16 & 3.73 & 0.07 & 3.33 & 0.052 \\
\hline MTV-T (per mL) & 1.026 & 0.02 & 1.037 & 0.002 & 1.033 & 0.003 \\
\hline Age (per y) & 1.079 & 0.10 & 1.039 & 0.23 & 1.042 & 0.13 \\
\hline MTV-T (per mL) & 1.019 & 0.06 & 1.037 & 0.001 & 1.029 & 0.004 \\
\hline Gender (female vs. male) & 0.561 & 0.55 & 3.437 & 0.19 & 1.438 & 0.57 \\
\hline MTV-T (per mL) & 1.020 & 0.07 & 1.033 & 0.003 & 1.028 & 0.006 \\
\hline Nodal status (N-positive vs. N-negative) & 1.086 & 0.93 & 0.995 & 1.0 & 1.183 & 0.80 \\
\hline MTV-T (per mL) & 1.024 & 0.13 & 1.045 & 0.005 & 1.035 & 0.005 \\
\hline HIV status (HIV-positive vs. HIV-negative) & 0.588 & 0.75 & 0.128 & 0.31 & 0.254 & 0.35 \\
\hline MTV-T (per $\mathrm{mL})$ & 1.028 & 0.02 & 1.034 & 0.002 & 1.031 & 0.003 \\
\hline MMC-based chemotherapy (yes vs. no) & 0.054 & 0.01 & 0.474 & 0.50 & 0.293 & 0.13 \\
\hline MTV-T (per mL) & 1.02 & 0.04 & 1.033 & 0.002 & 1.029 & 0.004 \\
\hline SUVmax & 0.987 & 0.87 & 0.970 & 0.65 & 0.992 & 0.88 \\
\hline MTV-T (per mL) & 1.023 & 0.03 & 1.032 & 0.002 & 1.028 & 0.005 \\
\hline SUVpeak & 0.911 & 0.42 & 1.026 & 0.61 & 1.012 & 0.79 \\
\hline MTV-T (per mL) & 1.020 & 0.04 & 1.033 & 0.001 & 1.028 & 0.004 \\
\hline SUVmean & 0.953 & 0.72 & 0.874 & 0.26 & 0.926 & 0.39 \\
\hline
\end{tabular}

\section{DISCUSSION}

This is the first study, to our knowledge, to demonstrate that pretherapy MTV-T is a significant prognostic marker for patients with anal cancer. We found that patients with a large pretherapy MTV-T had significantly worse PFS and EFS, independent of T classification, SUVmax, SUVpeak, nodal status, or sex. We did not find an association between SUVmax (or SUVpeak) and PFS, EFS, or OS.

The data regarding the prognostic value of baseline PET biomarkers in anal cancer are sparse. The report by Kidd et al. is currently the only study available in the literature (10). In that analysis, a tumor SUVmax greater than 5.6 was associated with worse DFS and predicted an incomplete response on posttreatment PET. The fact that SUVmax was not predictive of outcomes in our study is not surprising. First, it is possible that the smaller sample size of our study limited our ability to detect a significant difference. Second, SUVmax represents a single pixel that may be subject to a high degree of variability (noise bias) (23), thus making it a less reliable predictor of outcome across different studies. Last, SUVpeak is likely a more representative metric for the region of the tumor with the most metabolic activity. We found an improved association (though statistically insignificant) between PFS-EFS and SUVpeak, compared with PFS-EFS and SUVmax. More studies are clearly needed to further elucidate the role of PET as a prognostic marker in this disease site.

Two prior studies have highlighted the impact of posttreatment PET scans in patients with anal cancer. Schwarz et al. conducted a prospective study of 53 patients in which all underwent pretreatment and posttreatment PET scans (12). A partial metabolic response in the primary anal tumor as determined by the posttreatment PET scan predicted for worse
PFS. The PET-based response was more predictive than pretreatment tumor size or nodal status. Similarly, in the retrospective study by Day et al., a partial metabolic response on posttherapy PET was associated with worse OS and PFS; again, PET response was a stronger predictor than $\mathrm{T}$ or $\mathrm{N}$ classification (11). These results validate the observation in our study that PET biomarkers may be more valuable prognosticators than known clinical factors.

Although posttherapy PET scans appear to have a role in selecting patients for salvage therapy, the critical problem of identifying a high-risk patient group before treatment remains. In a cohort of over 600 patients from RTOG 98-11, Ajani et al. conclusively demonstrated that a tumor diameter greater than $5 \mathrm{~cm}$ (as well as male sex and nodal status) predicted poorer OS and DFS (5).

The importance of tumor size as demonstrated by Ajani et al. is evident in the fact that the $\mathrm{T}$ classification of anal tumors is based on size and not depth of invasion. A potential weakness of the parameter tumor size is that it may only be a surrogate for the underlying tumor burden. MTV-T defines tumor volume on the basis of the hypermetabolic activity within the tumor. In this sense, MTV-T is potentially a better biomarker than tumor size because it may reflect each tumor's intrinsic biology.

Currently, there is no uniform standard for the definition of MTV-T; it is calculated with absolute SUV thresholds, relative thresholds, or gradient-based methods. In a recent series of patients with head and neck cancer, Dibble et al. demonstrated excellent correlation between the gradientbased method and various relative thresholds (all Pearson $r$ correlation coefficients $>0.9$ ) (15). For each method used, there was a strong association between the calculated MTV and EFS. Thus, whereas the choice of segmentation method will affect the absolute volume that is measured, the con- 
sistency of the measurements should vary little as long as the same method is used in any study. Moving forward, prospective trials that assess the prognostic value of MTV need to clearly define the intensity method used to calculate MTV. However, the important point is that a growing number of studies continue to demonstrate that MTV is a strong prognostic marker in a variety of disease sites.

Limitations of our study include its retrospective nature and small sample size. The findings are therefore only hypothesis-generating and will need to be validated in a larger, ideally prospectively collected group of data. Also, the variability in timing and choice of surveillance imaging in our patients prevented a detailed analysis of posttherapy PET parameters. Likewise, the single-institution design of this study is limiting. At other institutions, differences in PET scanners, imaging protocols, and software analysis may affect the measurement and overall impact of PET parameters as biomarkers. Nonetheless, given the utility of PET in the staging and posttreatment evaluation of patients with anal cancer, we believe that future trials in this disease should incorporate PET into the study design. However, in such trials, systematic and strict protocols for PET will need to be established to minimize the potential variability in image analysis and follow-up schedules. Last, MTV has not been validated as a prognostic marker in a prospectively collected dataset in any disease site. Until further PET-based research is done in anal cancer and other malignancies, it will be challenging to define the optimal MTV cutoff points to riskstratify patients. Despite these limitations, attempts should be made to risk-stratify patients on the basis of MTV to help identify candidates for more aggressive therapies involving radiation or chemotherapy dose escalation or intensification.

\section{CONCLUSION}

Our study indicates that tumor burden, as assessed by pretreatment MTV-T, is prognostic for PFS, EFS, and OS in patients with SCC-AC. MTV-T was prognostic for PFS and EFS, independent of T classification or any of the other clinical parameters. SUV parameters did not correlate with survival or progression. Additional studies are needed to validate these findings and to establish the role of MTV-T as a tool to stratify patients for risk-adapted therapies in anal cancer.

\section{DISCLOSURE}

The costs of publication of this article were defrayed in part by the payment of page charges. Therefore, and solely to indicate this fact, this article is hereby marked "advertisement" in accordance with 18 USC section 1734. A generous donation was made by the Liaskas family in support of this work. This work was not funded by NIH or other grants. No other potential conflict of interest relevant to this article was reported.

\section{REFERENCES}

1. Epidermoid anal cancer: results from the UKCCCR randomised trial of radiotherapy alone versus radiotherapy, 5-fluorouracil, and mitomycin. UKCCCR
Anal Cancer Trial Working Party. UK Co-ordinating Committee on Cancer Research. Lancet. 1996;348:1049-1054.

2. Ajani JA, Winter KA, Gunderson LL, et al. Fluorouracil, mitomycin, and radiotherapy vs fluorouracil, cisplatin, and radiotherapy for carcinoma of the anal canal: a randomized controlled trial. JAMA. 2008;299:1914-1921.

3. Bartelink H, Roelofsen F, Eschwege F, et al. Concomitant radiotherapy and chemotherapy is superior to radiotherapy alone in the treatment of locally advanced anal cancer: results of a phase III randomized trial of the European Organization for Research and Treatment of Cancer Radiotherapy and Gastrointestinal Cooperative Groups. J Clin Oncol. 1997;15:2040-2049.

4. Flam M, John M, Pajak TF, et al. Role of mitomycin in combination with fluorouracil and radiotherapy, and of salvage chemoradiation in the definitive nonsurgical treatment of epidermoid carcinoma of the anal canal: results of a phase III randomized intergroup study. J Clin Oncol. 1996;14:2527-2539.

5. Ajani JA, Winter KA, Gunderson LL, et al. Prognostic factors derived from a prospective database dictate clinical biology of anal cancer: the intergroup trial (RTOG 98-11). Cancer. 2010;116:4007-4013.

6. Bannas P, Weber C, Adam G, et al. Contrast-enhanced $\left[{ }^{18} \mathrm{~F}\right]$ fluorodeoxyglucosepositron emission tomography/computed tomography for staging and radiotherapy planning in patients with anal cancer. Int J Radiat Oncol Biol Phys. 2011;81:445-451.

7. Cotter SE, Grigsby PW, Siegel BA, et al. FDG-PET/CT in the evaluation of anal carcinoma. Int J Radiat Oncol Biol Phys. 2006;65:720-725.

8. Nguyen BT, Joon DL, Khoo V, et al. Assessing the impact of FDG-PET in the management of anal cancer. Radiother Oncol. 2008;87:376-382.

9. Sveistrup J, Loft A, Berthelsen AK, Henriksen BM, Nielsen MB, Engelholm SA. Positron emission tomography/computed tomography in the staging and treatment of anal cancer. Int J Radiat Oncol Biol Phys. 2012;83:134-141.

10. Kidd EA, Dehdashti F, Siegel BA, Grigsby PW. Anal cancer maximum F-18 fluorodeoxyglucose uptake on positron emission tomography is correlated with prognosis. Radiother Oncol. 2010;95:288-291.

11. Day FL, Link E, Ngan S, et al. FDG-PET metabolic response predicts outcomes in anal cancer managed with chemoradiotherapy. Br J Cancer. 2011;105:498504.

12. Schwarz JK, Siegel BA, Dehdashti F, Myerson RJ, Fleshman JW, Grigsby PW. Tumor response and survival predicted by post-therapy FDG-PET/CT in anal cancer. Int J Radiat Oncol Biol Phys. 2008;71:180-186.

13. Lee P, Bazan JG, Lavori PW, et al. Metabolic tumor volume is an independent prognostic factor in patients treated definitively for non-small-cell lung cancer. Clin Lung Cancer. 2012;13:52-58.

14. Lee P, Weerasuriya DK, Lavori PW, et al. Metabolic tumor burden predicts for disease progression and death in lung cancer. Int J Radiat Oncol Biol Phys. 2007;69:328-333.

15. Dibble EH, Alvarez AC, Truong MT, Mercier G, Cook EF, Subramaniam RM. ${ }^{18} \mathrm{~F}$-FDG metabolic tumor volume and total glycolytic activity of oral cavity and oropharyngeal squamous cell cancer: adding value to clinical staging. $\mathrm{J} \mathrm{Nucl}$ Med. 2012;53:709-715.

16. La TH, Filion EJ, Turnbull BB, et al. Metabolic tumor volume predicts for recurrence and death in head-and-neck cancer. Int J Radiat Oncol Biol Phys. 2009;74:1335-1341.

17. Tang C, Murphy JD, Khong B, et al. Validation that metabolic tumor volume predicts outcome in head-and-neck cancer. Int J Radiat Oncol Biol Phys. 2012;83:1514-1520.

18. Jayachandran P, Pai RK, Quon A, et al. Postchemoradiotherapy positron emission tomography predicts pathologic response and survival in patients with esophageal cancer. Int J Radiat Oncol Biol Phys. 2012;84:471-477.

19. Schellenberg D, Quon A, Minn AY, et al. 18Fluorodeoxyglucose PET is prognostic of progression-free and overall survival in locally advanced pancreas cancer treated with stereotactic radiotherapy. Int J Radiat Oncol Biol Phys. 2010;77:1420-1425.

20. Bazan JG, Hara W, Hsu A, et al. Intensity-modulated radiation therapy versus conventional radiation therapy for squamous cell carcinoma of the anal canal. Cancer. 2011;117:3342-3351.

21. Graves EE, Quon A, Loo BW Jr. RT_Image: an open-source tool for investigating PET in radiation oncology. Technol Cancer Res Treat. 2007;6: 111-121.

22. Ciernik IF, Dizendorf E, Baumert BG, et al. Radiation treatment planning with an integrated positron emission and computer tomography (PET/CT): a feasibility study. Int J Radiat Oncol Biol Phys. 2003;57:853-863.

23. NCT00083083. Positron emission tomography pre- and post-treatment assessment for locally advanced non-small cell lung carcinoma. http://www.acrin.org/ Portals/0/Protocols/6668/ACRIN6668_Amend7_030210_ForONLINE.pdf.

24. Heagerty PJ, Lumley T, Pepe MS. Time-dependent ROC curves for censored survival data and a diagnostic marker. Biometrics. 2000;56:337-344. 